\title{
We are all choice architects: using behavioral economics to improve smoking cessation in primary care
}

\author{
Kevin Selby, MD MAS ${ }^{\top}$, Joachim Marti, $P h D^{7}$, and Marie-Anne Durand, MSC MPhil PhD \\ CPsychol ${ }^{1,2,3}$
}

${ }^{1}$ Center for Primary Care and Public Health (Unisanté), University of Lausanne, Lausanne, Switzerland; ${ }^{2}$ University of Toulouse III Paul Sabatier, Toulouse, France; ${ }^{3}$ Dartmouth University, Lebanon, New Hampshire, USA.

J Gen Intern Med 37(7):1783-5

DOI: $10.1007 / \mathrm{s} 11606-021-07322-2$

(C) The Author(s) under exclusive licence to Society of General Internal Medicine 2021

\begin{abstract}
A fter seeing Ms. V, a 55-year-old woman with hypertension and a 40-year history of smoking cigarettes, I scanned through her medical record. Late in her visit, I gingerly mentioned quitting smoking, like every year. She sighed: "It's bad for me, but I can't quit now. My teenage son is driving me crazy. Without a cigarette, I might kill him!" Now, I sigh. For years she has been "preparing to quit," but is never ready for action. She nods politely while I list the risks of smoking and rewards of quitting, but I never get past "assessing" her willingness to quit. What could I do differently?
\end{abstract}

\section{CURRENT APPROACH TO SMOKING CESSATION}

Discussions about smoking cessation in primary care begin, and often end, with a brief assessment of a patient's readiness to quit and a reminder that smoking is bad (Fig. 1). Deeply entrenched concepts from Prochaska and DiClemente's transtheoretical model of behavior change suggest we can reliably assess patients' readiness to adopt healthier behaviors with the question "Are you willing to give quitting a try?", reserving treatment discussions for those who are ready. ${ }^{1}$ Extensively applied to smoking cessation, the model postulates that smokers move through five stages of change: precontemplation (not ready), contemplation (getting ready), preparation (ready), action (adopting the healthier behavior), and maintenance (sustaining change and preventing a relapse). While intuitive, the approach is not supported by evidence ${ }^{2}$ and partly explains why tobacco use is undertreated as compared to other conditions. By asking permission, we stop

Received September 22, 2021

Accepted December 8, 2021

Published online January 11, 2022 short and most patients never hear about the evidencebased medications that can double or triple their chances of quitting. ${ }^{3}$ Nonetheless, the "stages of change" model remains influential in smoking cessation, as demonstrated by the "assess" step of the 5A's model (ask, advise, assess, assist, arrange) that is strongly advocated by primary care guidelines. ${ }^{1}$

When we ask patients if they are ready, today, to discuss quitting, we are asking them to "opt in" to a discussion about quitting, before mentioning potential treatments. Behavioral economics tells us that asking patients to opt in to difficult conversations about behavior change encounters numerous cognitive biases. We all struggle to look beyond immediate pleasure for long-term benefits (present bias). We overestimate the effect of giving up what we have (loss aversion bias). When overwhelmed by multiple difficult decisions, we take no action (status quo bias). Research with people who have quit smoking suggests decisions are made impulsively without passing predictably through rational stages. ${ }^{4}$ Self-reported "readiness" to quit is a poor predictor of who will accept a smoking aid prescription and make a quit attempt. ${ }^{5}$ Randomized trials have demonstrated that many patients who were not planning to quit will nonetheless accept and use a prescription for a quit aid. Physicians could circumvent the above biases and reach more smokers by modifying their approach to smoking cessation.

\section{IMPROVING THE CHOICE ARCHITECTURE OF SMOKING CESSATION}

We do not need to look far for a different model. We offer most treatments as the default choice. If a diabetic patient's blood sugars are consistently above target despite metformin, we prescribe more intensive treatment unless they refuse (opt out). Default choices are extremely powerful; near the end of life, $77 \%$ of patients choose comfort-oriented care when presented that as the default, as opposed to only $43 \%$ of patients presented life-extending care as the default. ${ }^{6}$ The difference between groups is not explained by stated preferences, but by inertia around the default. ${ }^{7}$

When prescribing a new treatment, the default should not be to assign a medication, but to encourage patients to make an 


\section{A) Current approach (5 As)}

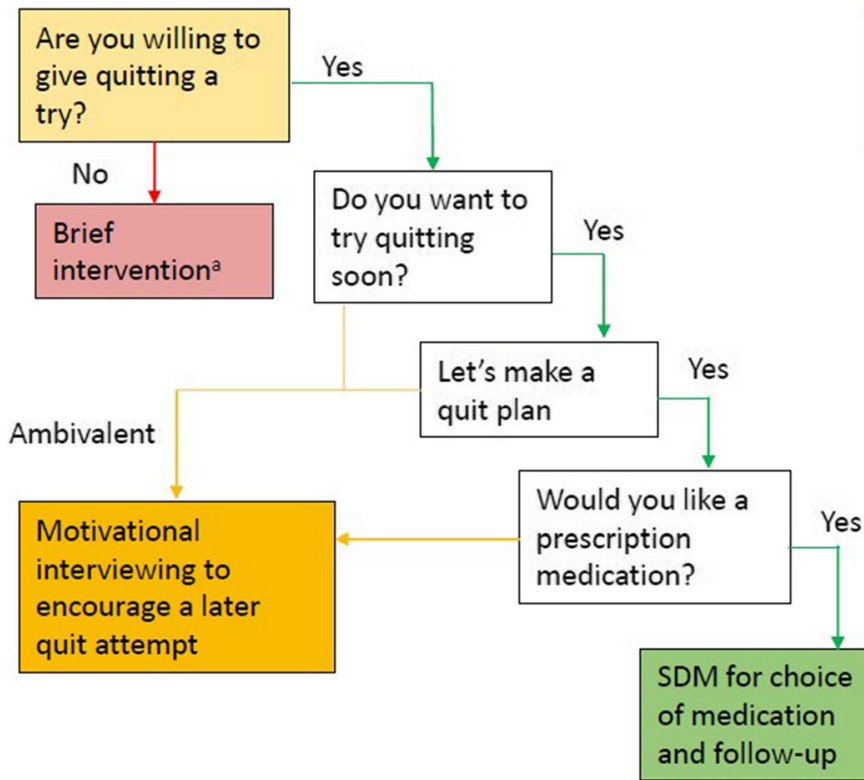

B) Default choice and shared decision making (SDM)

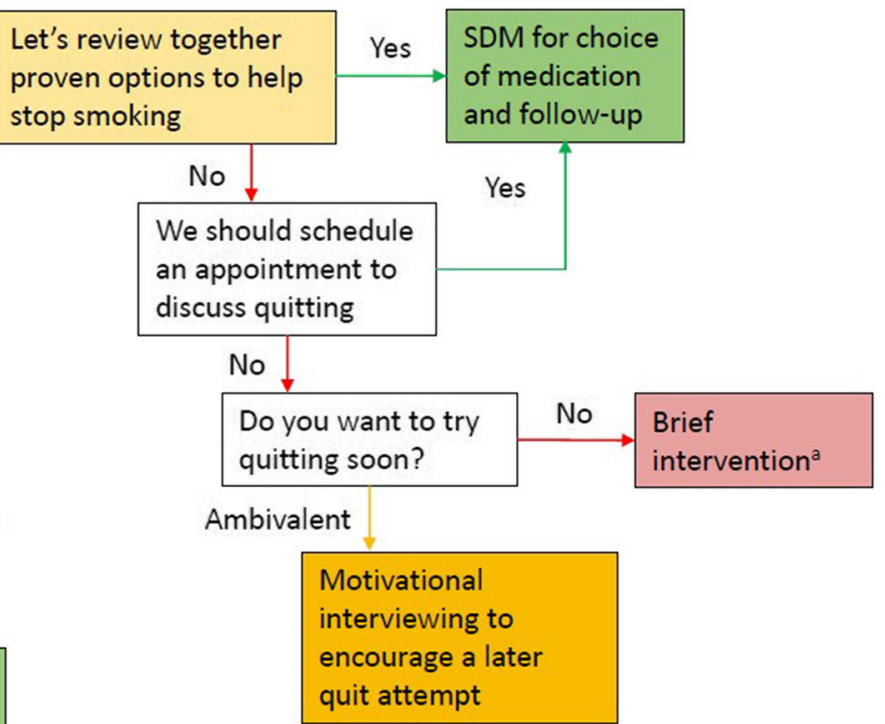

Figure 1 Comparison of the decision architecture of $A$ our current recommended approach to smoking cessation in primary care and $B$ a new

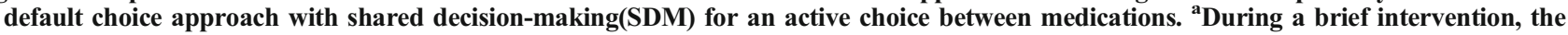
clinician takes approximately 2 minutes to clearly advise the patient to quit smoking and mention resources available to quit smoking.

active choice between available medications using shared decision-making. Using shared decision-making principles, the discussion of individual preferences is then focused on an active choice between options. With an active choice, patients explicitly choose their own treatment; active choices, rather than passive ones, improve outcomes even further. ${ }^{7}$

For smoking cessation, discussions could begin by offering all current smokers a menu of treatments for smoking cessation, regardless of their stated readiness to quit (Fig. 1). Current smokers would therefore "opt out" of choosing a treatment. The "refer" step of the shorter, more proactive AAR model (ask, advise, refer) goes in this direction, but is typically implemented by giving patients pamphlets or links to outside resources. ${ }^{1}$ Ideally, patients should choose between treatment options directly prescribed by their provider. Trying a treatment should be the easiest option.

\section{BEHAVIORAL ECONOMICS IN OFFICE-BASED PREVENTION}

Choice architecture examines how information layout, range, order, and extent of options displayed can shape decisionmaking. While much has been written about choice architecture as a means of modifying physician behavior or improving public health interventions, we rarely discuss how primary care providers already use choice architecture intuitively to influence patient behavior. In the case of smoking cessation, our intuition to only discuss treatments with smokers who opt in is misguided.

Teaching providers to understand how they already use choice architecture could allow them avoid cognitive biases, help their patients make better choices, and lead to more impactful health prevention recommendations. By understanding principles of behavioral economics, providers could present default choices when there is a single, preferred treatment and active choices when there is equipoise between reasonable alternatives. Patients can still refuse the default choice, but evidence suggests more of them will accept.

With this teaching, we could present prevention activities like colorectal and cervical cancer screening as the default choice, using positive framing to underline the benefits of screening, and engaging in shared decision-making to choose between testing modalities. Weak recommendations like prostate cancer screening or breast cancer screening between the ages of 40 and 49 may default to no screening after presenting evidence-based information about the potential benefits and risks of each situation.

Default options may be seen as paternalistic or not patient centered. However, once we recognize that we already use aspects of behavioral economics like framing and nudges, improved knowledge of choice architecture allows us use these tools correctly. Decision architecture provides a powerful tool to encourage healthy behaviors. Because time is so limited in busy primary care visits, even subtle use of suboptimal decision architecture can lead to the underuse of important treatments, like medications for smoking cessation. It is essential that strongly recommended, life-saving treatments be the easy choice.

Returning to Ms. V, the next time I see her, I will enter directly into an active choice between treatment options, instead of returning to our discussion about the right time to try quitting. I will use a conversation aid to compare available 
treatment options and highlight differences regarding side effects and efficacy. Varenicline is highly efficacious but may have side effects; combined long- and short-acting nicotine replacement therapy is efficacious but expensive in Switzerland; an electronic cigarette with nicotine is likely effective, but many patients continue using it longer than 3 months, raising concerns about addictiveness and unknown long-term safety. She might try varenicline, despite doubts she can really quit. I'm glad, because varenicline also benefits smokers who aren't willing to stop abruptly.

Closer examination of our decision architecture could improve choices for preventive health. A clear example is the direct discussion of treatments for smoking cessation without assessing patients' readiness to quit; by encouraging an active choice, we might increase the number of patients using proven quit aids. We cannot make quitting easy, but at least we will not wait 10 years for the perfect moment to try.

Acknowledgements: The authors would like to thank Professor Catherine Maclean, Professor Ivan Berlin, and Doctor Samuel Edwards for their critical re-reading of the manuscript.

Corresponding Author: Kevin Selby, MD MAS; Center for Primary Care and Public Health (Unisanté), University of Lausanne, Lausanne, Switzerland (e-mail: kevin.selby@unisante.ch).

Funding This work is funded in part by the Swiss Tobacco Prevention Fund. Dr. Selby receives salary support from the Leenaards Foundation.

\section{Declarations:}

Conflict of Interest: Dr. Selby has developed a freely available online application to help with shared decision-making in smoking cessation (www.howtoquit.ch). Marie-Anne Durand has contributed to the development of Option Grid conversation aids, which are licensed to EBSCO Health. She receives consulting income from EBSCO Health and may receive royalties.

\section{REFERENCES}

1. Agency for Healthcare Research and Quality. Treating Tobacco Use and Dependence: 2008 Update. 2008; https://www.ahrq.gov/prevention/ guidelines/tobacco/index.html. Accessed February 23, 2021.

2. Cahill K, Lancaster T, Green N. Stage-based interventions for smoking cessation. Cochrane Database of Systematic Reviews. 2010(11):CD004492.

3. Cohen BP, McGinnis S, Salsberg E. Physician Behavior and Practice Patterns Related to Smoking Cessation. Washington, D.C.: Association of American Medical Colleges;2007.

4. Ferguson SG, Shiffman S, Gitchell JG, Sembower MA, West R. Unplanned quit attempts-results from a U.S. sample of smokers and ex-smokers. Nicotine Tob Res. 2009;11(7):827-832.

5. Danan ER, Joseph AM, Sherman SE, et al. Does Motivation Matter? Analysis of a Randomized Trial of Proactive Outreach to VA Smokers. J Gen Intern Med. 2016;31(8):878-887.

6. Halpern SD, Loewenstein G, Volpp KG, et al. Default Options In Advance Directives Influence How Patients Set Goals For End-Of-Life Care. Health Affairs. 2013;32(2):408-417

7. Beshears J, Choi JJ, Laibson D, Madrian BC. Active Choice, Implicit Defaults, and the Incentive to Choose. National Bureau of Economic Research Working Paper Series. 2019;24573.

Publisher's Note: Springer Nature remains neutral with regard to jurisdictional claims in published maps and institutional affiliations. 